\title{
THE BOSS EMISSION-LINE LENS SURVEY. IV. SMOOTH LENS MODELS for the BELLS GALLERY SAMPLE
}

DOI:

10.3847/1538-4357/833/2/264

Link to publication record in Manchester Research Explorer

\section{Citation for published version (APA):}

Shu, Y., Bolton, A. S., Mao, S., Kochanek, C. S., Pérez-Fournon, I., Oguri, M., Montero-Dorta, A. D., Cornachione, M. A., Marques-Chaves, R., Zheng, Z., Brownstein, J. R., \& Ménard, B. (2016). THE BOSS EMISSION-LINE LENS SURVEY. IV. SMOOTH LENS MODELS for the BELLS GALLERY SAMPLE. Astrophysical Journal, 833(2), [264]. https://doi.org/10.3847/1538-4357/833/2/264

\section{Published in:}

Astrophysical Journal

\section{Citing this paper}

Please note that where the full-text provided on Manchester Research Explorer is the Author Accepted Manuscript or Proof version this may differ from the final Published version. If citing, it is advised that you check and use the publisher's definitive version.

\section{General rights}

Copyright and moral rights for the publications made accessible in the Research Explorer are retained by the authors and/or other copyright owners and it is a condition of accessing publications that users recognise and abide by the legal requirements associated with these rights.

\section{Takedown policy}

If you believe that this document breaches copyright please refer to the University of Manchester's Takedown Procedures [http://man.ac.uk/04Y6Bo] or contact uml.scholarlycommunications@manchester.ac.uk providing relevant details, so we can investigate your claim.

\section{OPEN ACCESS}




\title{
THE BOSS EMISSION-LINE LENS SURVEY. IV. SMOOTH LENS MODELS FOR THE BELLS GALLERY SAMPLE*
}

\author{
Yiping Shu ${ }^{1}$, Adam S. Bolton ${ }^{2,3}$, Shude Mao ${ }^{4,1,5}$, Christopher S. KochaneK ${ }^{6}$, Ismael Pérez-Fournon ${ }^{7,8}$, \\ Masamune Oguri ${ }^{9}, 10,11$, Antonio D. Montero-Dorta ${ }^{2}$, Matthew A. Cornachione ${ }^{2}$, Rui Marques-Chaves ${ }^{7,8}$, \\ Zheng Zheng ${ }^{2}$, Joel R. BRownstein ${ }^{2}$, ANd Brice Ménard ${ }^{12}$ \\ ${ }^{1}$ National Astronomical Observatories, Chinese Academy of Sciences, 20A Datun Road, Chaoyang District, Beijing 100012, China; yiping.shu@nao.cas.cn \\ ${ }^{2}$ Department of Physics and Astronomy, University of Utah, 115 South 1400 East, Salt Lake City, UT 84112, USA \\ ${ }^{3}$ National Optical Astronomy Observatory, 950 N. Cherry Avenue, Tucson, AZ 85719, USA \\ ${ }^{4}$ Physics Department and Tsinghua Centre for Astrophysics, Tsinghua University, Beijing 100084, China \\ ${ }^{5}$ Jodrell Bank Centre for Astrophysics, School of Physics and Astronomy, The University of Manchester, Oxford Road, Manchester M13 9PL, UK \\ ${ }^{6}$ Department of Astronomy and Center for Cosmology and Astroparticle Physics, Ohio State University, Columbus, OH 43210, USA \\ ${ }^{7}$ Instituto de Astrofísica de Canarias, C/Vía Láctea, s/n, E-38205 San Cristóbal de La Laguna, Tenerife, Spain \\ ${ }^{8}$ Universidad de La Laguna, Dpto. Astrofísica, E-38206 La Laguna, Tenerife, Spain \\ ${ }^{9}$ Research Center for the Early Universe, University of Tokyo, 7-3-1 Hongo, Bunkyo-ku, Tokyo 113-0033, Japan \\ ${ }^{10}$ Department of Physics, University of Tokyo, 7-3-1 Hongo, Bunkyo-ku, Tokyo 113-0033, Japan \\ ${ }^{11}$ Kavli Institute for the Physics and Mathematics of the Universe (Kavli IPMU, WPI), University of Tokyo, Chiba 277-8583, Japan \\ ${ }^{12}$ Department of Physics and Astronomy, Johns Hopkins University, Baltimore, MD 21218, USA \\ Received 2016 July 20; revised 2016 October 23; accepted 2016 November 4; published 2016 December 21
}

\begin{abstract}
We present Hubble Space Telescope F606W-band imaging observations of 21 galaxy-Ly $\alpha$ emitter lens candidates in the Baryon Oscillation Spectroscopic Survey Emission-Line Lens Survey (BELLS) for the GALaxy-Lya EmitteR sYstems (BELLS GALLERY) survey. Seventeen systems are confirmed to be definite lenses with unambiguous evidence of multiple imaging. The lenses are primarily massive early-type galaxies (ETGs) at redshifts of approximately 0.55 , while the lensed sources are Ly $\alpha$ emitters (LAEs) at redshifts from two to three. Although most of the lens systems are well fit by smooth lens models consisting of singular isothermal ellipsoids in an external shear field, a thorough exploration of dark substructures in the lens galaxies is required. The Einstein radii of the BELLS GALLERY lenses are, on average, 60\% larger than those of the BELLS lenses because of the much higher source redshifts. This will allow for a detailed investigation of the radius evolution of the mass profile in ETGs. With the aid of the average $\sim 13 \times$ lensing magnification, the LAEs are frequently resolved into individual star-forming knots with a wide range of properties. They have characteristic sizes from less than $100 \mathrm{pc}$ to several kiloparsecs, rest-frame far-UV apparent $\mathrm{AB}$ magnitudes from 29.6 to 24.2 , and typical projected separations of $500 \mathrm{pc}$ to $2 \mathrm{kpc}$.
\end{abstract}

Key words: dark matter - galaxies: elliptical and lenticular, cD - gravitational lensing: strong techniques: image processing

\section{INTRODUCTION}

Strong gravitational lenses are sensitive to the total mass distribution regardless of its form. This allows for the extensive use of lenses as a powerful probe of lens galaxies including stars, dark matter, and dark substructures (e.g., Kochanek 1995; Keeton et al. 1998; Rusin et al. 2003; Bolton et al. 2008a, 2008b; Auger et al. 2010; Vegetti et al. 2010, 2012; Dutton et al. 2011; Spiniello et al. 2011; Barnabè et al. 2012; Bolton et al. 2012b; Brewer et al. 2012; Brownstein et al. 2012; Fadely \& Keeton 2012; MacLeod et al. 2013; Sonnenfeld et al. 2013; Oguri et al. 2014; Nierenberg et al. 2014; Shu et al. 2015, 2016b; Hezaveh et al. 2016; Inoue et al. 2016). Furthermore, the magnification effect, by which the apparent size and total flux of the lensed source increase up to factors of tens, makes strong gravitational lensing a natural "magnifier" for studies of faint high-redshift objects (e.g., Bolton et al. 2006b; Quider et al. 2009; Christensen et al. 2012; Muzzin et al. 2012; Bussmann et al. 2013; Stark et al. 2015; Karman et al. 2016; Shu et al. 2016b; Spilker et al. 2016).

* Based on observations made with the NASA/ESA Hubble Space Telescope, obtained from the Data Archive at the Space Telescope Science Institute, which is operated by AURA, Inc., under NASA contract NAS 5-26555. These observations are associated with program \#14189.
Bolton et al. (2004) developed a novel technique to efficiently select galaxy-scale strong gravitational lenses by searching along the line of sight toward a potential lens for multiple emission lines from a common redshift beyond the redshift of the foreground object. Follow-up high-resolution Hubble Space Telescope (HST) imaging observations and associated lens models can then confirm the lensing nature of the systems. The application of this spectroscopic-selection and $H S T$-observation strategy to the enormous database of galaxy spectra in the Sloan Digital Sky Survey (SDSS; York et al. 2000) and the Baryon Oscillation Spectroscopic Survey (BOSS; Dawson et al. 2013) of the Sloan Digital Sky SurveyIII (SDSS-III; Eisenstein et al. 2011) has resulted in four dedicated surveys: the Sloan Lens ACS (SLACS; Bolton et al. 2006a) survey, the Sloan WFC Edge-on Late-type Lens Survey (SWELLS; Treu et al. 2011), the SLACS for the Masses (S4TM; Shu et al. 2015) survey, and the BOSS Emission-Line Lens Survey (BELLS; Brownstein et al. 2012). Over 150 grade-A galaxy lenses with clear and convincing evidence for multiple imaging have been discovered from the four surveys leading to a broad range of scientific discoveries (e.g., Koopmans et al. 2006; Treu et al. 2006, 2009; Gavazzi et al. 2007; Bolton et al. 2008a, 2008b, 2012b; Czoske et al. 2008; Gavazzi et al. 2008; Auger et al. 2009, 2010; Barnabè 
et al. 2009, 2011, 2012; Grillo et al. 2010; Dutton et al. 2011, 2013; Newton et al. 2011; Brewer et al. 2012, 2014; Shu et al. 2015).

We initiated the BELLS for GAlaxy-Ly $\alpha$ EmitteR sYstems (BELLS GALLERY) survey in 2015 to search for low-mass, dark substructures in lens galaxies by utilizing the intrinsic compactness of high-redshift lensed Ly $\alpha$ emitters (LAEs). The galaxy-LAE strong lens candidate systems are spectroscopically selected from almost 1.5 million galaxy spectra in the final data release (DR12) of the BOSS survey. Cuts on the detection significance and apparent flux and profile of the detected emission line were applied to the parent sample of 187 candidates to select the 21 highest-quality candidates that compose the BELLS GALLERY sample. The foreground lenses are classified as massive early-type galaxies (ETGs) with a median redshift of 0.55 as determined by the BOSS spectroscopic data. The background sources are LAEs at redshifts from 2 to 3 with a median redshift of 2.5. Detailed descriptions of the selection algorithm and properties of the sample are presented in a previous paper (BELLS-III; Shu et al. 2016a).

This paper, as the fourth in a series about the BELLS survey, presents the follow-up imaging data from the recently finished $H S T$ observations along with lens models constructed from the data. It is organized as follows. Section 2 provides the HST observational data and the derived photometric properties of the lens galaxies. Smooth lens models (without any substructure) are presented in Section 3. Discussion and conclusions are given in Sections 4 and 5, respectively. Throughout the paper, we adopt a fiducial cosmological model with $\Omega_{\mathrm{m}}=0.274$, $\Omega_{\Lambda}=0.726$, and $H_{0}=70 \mathrm{~km} \mathrm{~s}^{-1} \mathrm{Mpc}^{-1}$ (WMAP7, Komatsu et al. 2011).

\section{HST DATA}

The HST follow-up observations of the 21 BELLS GALLERY candidates started in 2015 November under HST Cycle 23 program ID 14189 (PI: A. Bolton) and finished in 2016 May. For each candidate, 4 sub-exposures of approximately $630 \mathrm{~s}$ each were taken within a single $H S T$ orbit using the $V$-band F606W filter on the Wide Field Camera 3 (WFC3). The individual flat-fielded (FLT) sub-exposure files for each system were fully reduced, rectified onto uniform pixels of 0 !"04, and combined using the custom-built and extensively tested reduction pipeline, ACSPROC, following the procedures described in Bolton et al. (2008a), Brownstein et al. (2012), and Shu et al. (2015). We modified ACSPROC for the shift from the ACS/WFC to the WFC3/UVIS camera. The empirical pointspread function (PSF) was generated by the Tiny Tim tool (Krist 1993). Pixel count errors are rescaled following Shu et al. $(2015,2016 b)$ to correct for possible error correlations created by imaging resampling during the data reduction process. The mean pixel count error after the rescaling is $\sim 0.005$ electrons per second per pixel ${ }^{2}$.

Figure 1 shows a mosaic of the fully reduced images of the 21 candidate systems in the BELLS GALLERY survey. The cutouts are centered on the R.A. and decl. of the lens galaxy as determined by the BOSS survey. For all systems but J091859.21+510452.5 (the "SDSS" is omitted to save the space), the cutout centers are coincident with the lens galaxy centers. The two lens components in J091859.21+510452.5 are not resolved by BOSS, and therefore its cutout is centered between the two lens components. For the background LAEs, the HST F606W filter covers their rest-frame far-ultraviolet (UV) emission. We manually generate feature masks that enclose the regions that are suspected to be lensed features (red dots). Junk masks for contaminating structures are also generated in the same fashion and outlined by the green dots. The pixel count errors within the junk masks are set to infinity so that they do not affect the actual fitting. To be compatible with the previous SLACS, BELLS, and S4TM surveys, we carry out a smooth $b$-spline fit to the foreground-light distribution of each system following Bolton et al. (2006a, 2008a). Besides the b-spline model, an elliptical Sérsic model (Sérsic 1963) is also used for the foreground-light subtraction. As will be explained in the following section, the Sérsic fit is performed simultaneously with the lens modeling to reduce potential systematics. The b-spline-subtracted residual image of every candidate system is inspected visually for lensed features, and a classification code, which characterizes the lens morphology, multiplicity, and status, is assigned accordingly.

Table 1 summarizes the properties of the BELLS GALLERY sample measured by the BOSS data reduction pipeline (Bolton et al. 2012a), the Sérsic fit parameters based on the HST imaging data, and the classification results. Although not directly comparable because of differences in data quality and assumed light profile, the effective radii measured from the BOSS and HST data are in general agreement. The axis ratio distribution measured from HST data is consistent with that for ETGs in general (e.g., Hao et al. 2006). In terms of lens status, 17 of the 21 candidate systems are confirmed to be grade-A galaxy-LAE lenses with unambiguous lensed features. This includes 8 lenses with extended arcs, 3 with quadruple images, and 6 with double images. Three systems, J005409.97+294450.8, J151641.22 +495440.7 , and $\mathrm{J} 152926.41+401548.8$, are simply non-lenses with singly imaged sources. The remaining system, J224505.93 +004018.3 , shows complex structures that are too hard to interpret based on this single-band imaging data, so we conservatively consider this system a "maybe" for the moment. Further multi-band imaging data might reveal the true status of this system. The success rate of $81 \%$ (17 lenses from 21 candidates) is much higher than those for previous SLACS, BELLS, and S4TM surveys based on similar selection techniques. This is presumably due to the much more stringent selection cuts applied to the BELLS GALLERY sample and the inclusion of two systems, J020121.39+322829.6 and J075523.52+344539.5, that do not meet the selection thresholds but show definite evidence for strong-lensing features in their color-composite SDSS images. There remain another 166 candidate systems in the parent sample with high signal-to-noise ratio detections of "anomalous" emission lines. Considering the typical 50\% success rate in the previous surveys, we expect another $\sim 70$ galaxy-LAE lens systems from future follow-up observations of the remaining sample.

\section{SMOOTH LENS MODELS}

\subsection{Methodology}

Building on our previous works (Bolton et al. 2008a; Brownstein et al. 2012; Shu et al. 2015, 2016b), we develop an open source lens modeling tool lfit_gui with a graphical user interface (GUI). In this subsection, we use the BELLS GALLERY lenses to demonstrate the settings and functions of lfit_gui. 

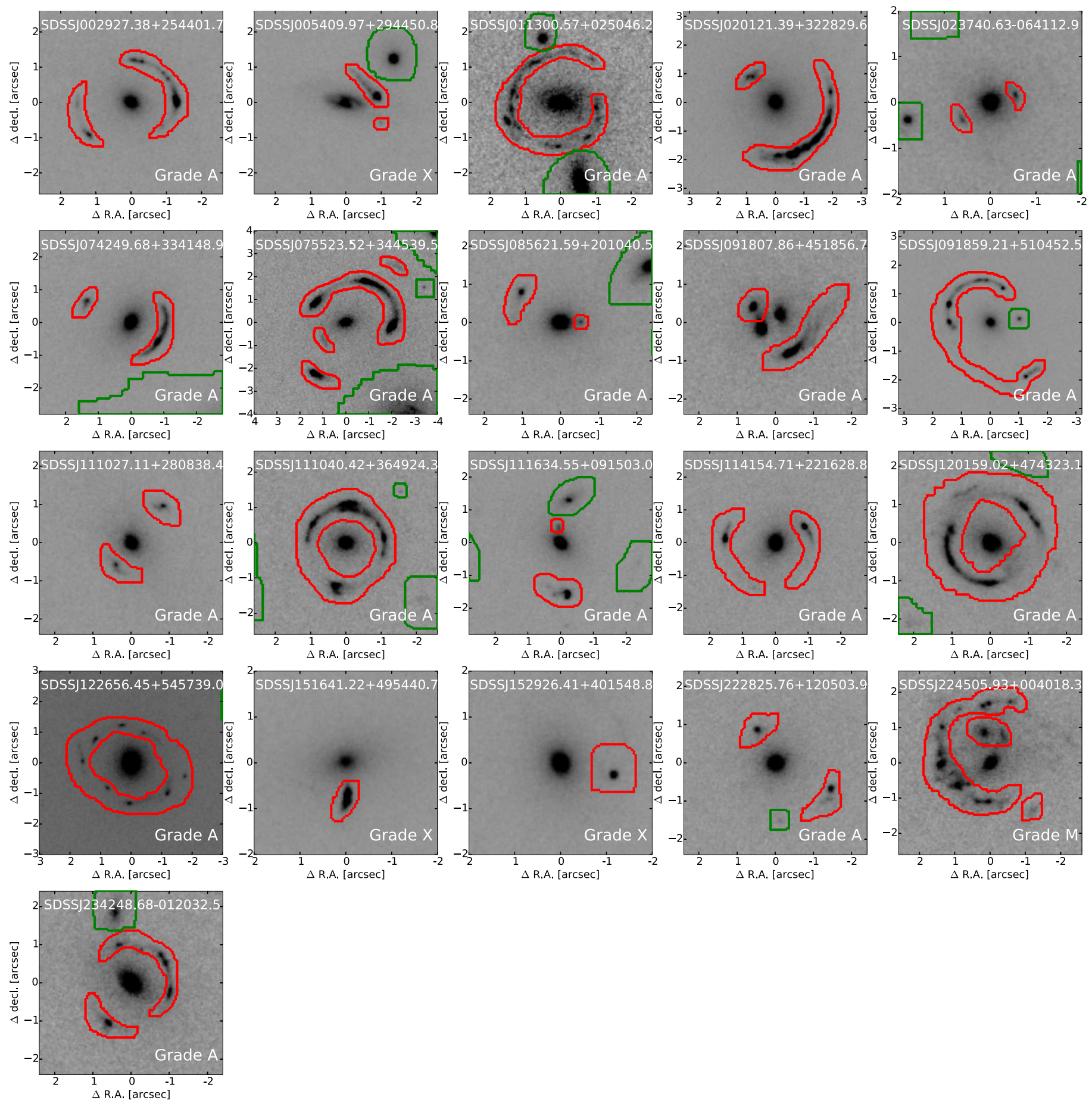

Figure 1. Mosaic of the HST F606W-band images of 21 BELLS GALLERY candidate systems. The images are orientated such that north is up and east is to the left. The axis labels provide offsets in R.A. and decl., respectively. The regions bounded by the red dots are regions that are thought to be related to the background source, while the green dots outline regions with contaminating structures. All systems but J005409.97+294450.8, J151641.22+495440.7, J152926.41+401548.8, and J224505.93+004018.3 are clearly multiply imaged lenses (Grade A). J005409.97+294450.8, J151641.22+495440.7, and J152926.41+401548.8 are only singly imaged (Grade X), while J224505.93+004018.3 is too complex to classify (Grade M).

\subsubsection{Foreground-light Subtraction}

Foreground-light removal is a crucial step in gravitational lens modeling when the emission from the foreground lens is high enough to hinder the correct interpretation of the lensed emission. Being massive elliptical galaxies, the BELLS GALLERY lenses contribute substantial fractions of the observed optical emission. One commonly used approach is to perform the foreground-light subtraction separately prior to the lens modeling (e.g., Moustakas et al. 2007; Bolton et al. 2008a; Suyu \& Halkola 2010; Newton et al. 2011; Brownstein et al. 2012; Sonnenfeld et al. 2013; Shu et al. 2015). However, this can lead to two problems: (1) automatic adjustments are not possible if the lensing and junk features are masked inappropriately, which can affect the foreground-light fitting; (2) the pixel count errors estimated for the full data might not be appropriate for the foreground-subtracted residual, and 
Table 1

Selected Properties of the BELLS GALLERY Sample

\begin{tabular}{|c|c|c|c|c|c|c|c|c|}
\hline \multirow{2}{*}{ Target } & \multicolumn{4}{|c|}{ BOSS } & \multicolumn{3}{|c|}{ HST F606W } & \multirow{2}{*}{$\begin{array}{l}\text { Classification } \\
\text { (9) }\end{array}$} \\
\hline & $\begin{array}{l}z_{L} \\
\text { (2) }\end{array}$ & $\begin{array}{l}z_{S} \\
(3)\end{array}$ & $\begin{array}{c}\sigma_{\mathrm{BOSS}} \\
\left(\mathrm{km} \mathrm{s}^{-1}\right) \\
(4)\end{array}$ & $\begin{array}{c}R_{\mathrm{dev}} \\
(\operatorname{arcsec}) \\
\quad(5)\end{array}$ & $\begin{array}{c}R_{\text {eff }} \\
(\operatorname{arcsec}) \\
(6)\end{array}$ & $\begin{array}{l}q \\
\text { (7) }\end{array}$ & $\begin{array}{c}\text { P.A. } \\
\text { (degree) } \\
\text { (8) }\end{array}$ & \\
\hline SDSSJ002927.38+254401.7 & 0.5869 & 2.4504 & $241 \pm 45$ & $1.43 \pm 0.71$ & $0.49 \pm 0.01$ & $0.825 \pm 0.006$ & $52.2 \pm 1.1$ & E-S-A \\
\hline SDSSJ005409.97+294450.8 & 0.4488 & 2.7176 & $177 \pm 58$ & $1.31 \pm 0.37$ & $0.39 \pm 0.01$ & $0.457 \pm 0.007$ & $81.6 \pm 0.5$ & L-S-X \\
\hline SDSSJ011300.57+025046.2 & 0.6230 & 2.6088 & $850 \pm-1$ & $2.80 \pm 1.26$ & $1.84 \pm 0.22$ & $0.645 \pm 0.008$ & $79.3 \pm 0.8$ & E-M-A \\
\hline SDSSJ020121.39+322829.6 & 0.3957 & 2.8209 & $256 \pm 20$ & $2.60 \pm 0.38$ & $2.32 \pm 0.16$ & $0.882 \pm 0.004$ & $21.1 \pm 1.1$ & E-S-A \\
\hline SDSSJ023740.63-064112.9 & 0.4859 & 2.2491 & $290 \pm 89$ & $1.05 \pm 0.45$ & $1.05 \pm 0.09$ & $0.980 \pm 0.008$ & $109.0 \pm 11.1$ & E-S-A \\
\hline SDSSJ074249.68+334148.9 & 0.4936 & 2.3633 & $218 \pm 28$ & $1.07 \pm 0.32$ & $0.89 \pm 0.03$ & $0.717 \pm 0.004$ & $148.3 \pm 0.4$ & E-S-A \\
\hline SDSSJ075523.52+344539.5 & 0.7224 & 2.6347 & $272 \pm 52$ & $0.27 \pm 0.72$ & $2.89 \pm 0.47$ & $0.602 \pm 0.007$ & $102.4 \pm 0.6$ & E-S-A \\
\hline SDSSJ085621.59+201040.5 & 0.5074 & 2.2335 & $334 \pm 54$ & $1.15 \pm 0.42$ & $0.51 \pm 0.01$ & $0.795 \pm 0.005$ & $91.9 \pm 0.8$ & E-S-A \\
\hline SDSSJ091807.86+451856.7 & 0.5238 & 2.3440 & $119 \pm 61$ & $2.08 \pm 1.16$ & $2.22 \pm 0.73$ & $0.807 \pm 0.016$ & $58.6 \pm 2.5$ & E-M-A \\
\hline SDSSJ091859.21+510452.5 & 0.5811 & 2.4030 & $298 \pm 49$ & $1.89 \pm 0.77$ & $0.57 \pm 0.02$ & $0.880 \pm 0.008$ & $39.1 \pm 2.1$ & E-S-A \\
\hline SDSSJ111027.11+280838.4 & 0.6073 & 2.3999 & $191 \pm 39$ & $0.40 \pm 0.23$ & $1.45 \pm 0.16$ & $0.762 \pm 0.006$ & $31.4 \pm 0.8$ & E-S-A \\
\hline SDSSJ111040.42+364924.4 & 0.7330 & 2.5024 & $531 \pm 165$ & $0.88 \pm 0.40$ & $0.39 \pm 0.01$ & $0.779 \pm 0.008$ & $88.4 \pm 1.1$ & E-S-A \\
\hline SDSSJ111634.55+091503.0 & 0.5501 & 2.4536 & $274 \pm 55$ & $0.95 \pm 0.35$ & $0.98 \pm 0.06$ & $0.690 \pm 0.005$ & $41.0 \pm 0.5$ & E-S-A \\
\hline SDSSJ114154.71+221628.8 & 0.5858 & 2.7624 & $285 \pm 44$ & $0.63 \pm 0.25$ & $0.44 \pm 0.01$ & $0.801 \pm 0.005$ & $157.6 \pm 0.9$ & E-S-A \\
\hline SDSSJ120159.02+474323.2 & 0.5628 & 2.1258 & $239 \pm 43$ & $1.83 \pm 0.45$ & $0.48 \pm 0.01$ & $0.738 \pm 0.006$ & $57.9 \pm 0.7$ & E-S-A \\
\hline SDSSJ122656.45+545739.0 & 0.4980 & 2.7322 & $248 \pm 26$ & $1.12 \pm 0.20$ & $0.56 \pm 0.01$ & $0.827 \pm 0.003$ & $2.0 \pm 0.6$ & E-S-A \\
\hline SDSSJ151641.22+495440.7 & 0.5479 & 2.8723 & $226 \pm 40$ & $1.54 \pm 0.39$ & $1.71 \pm 0.07$ & $0.618 \pm 0.004$ & $107.7 \pm 0.3$ & E-S-X \\
\hline SDSSJ152926.41+401548.8 & 0.5308 & 2.7920 & $283 \pm 33$ & $1.56 \pm 0.52$ & $1.65 \pm 0.11$ & $0.798 \pm 0.004$ & $18.6 \pm 0.7$ & E-S-X \\
\hline SDSSJ222825.76+120503.9 & 0.5305 & 2.8324 & $255 \pm 50$ & $0.82 \pm 0.39$ & $0.53 \pm 0.02$ & $0.951 \pm 0.007$ & $106.3 \pm 4.3$ & E-S-A \\
\hline SDSSJ224505.93+004018.3 & 0.7021 & 2.5413 & $64 \pm 44$ & $1.84 \pm 0.65$ & $2.69 \pm 0.51$ & $0.687 \pm 0.009$ & $125.5 \pm 1.0$ & E-S-M \\
\hline SDSSJ234248.68-012032.5 & 0.5270 & 2.2649 & $274 \pm 43$ & $1.32 \pm 0.66$ & $1.75 \pm 0.19$ & $0.682 \pm 0.006$ & $44.9 \pm 0.6$ & E-S-A \\
\hline
\end{tabular}

Note. Column 1 is the SDSS system name in terms of the truncated J2000 R.A. and decl. in the format HHMMSS.ss \pm DDMMSS.s. Columns 2 and 3 are the redshifts of the foreground lens and the background LAE inferred from the BOSS spectrum. Column 4 is the velocity dispersion calculated from the velocitydispersion likelihood function using restricted stellar eigenspectra with redshift-error marginalization, as described in Shu et al. (2012). The best-fit velocity dispersion for SDSS J011300.57+025046.2, $850 \mathrm{~km} \mathrm{~s}^{-1}$, is at the maximum dispersion value tested and is therefore unreliable. The associated error is set to -1 as a warning flag. Column 5 is the BOSS $r$-band de Vaucouleurs fit effective radius (in the intermediate axis convention) of the foreground lens. Columns 6-8 are the effective radius (in the intermediate axis convention), minor-to-major axis ratio, and major-axis position angle of the lens galaxy with respect to the north inferred from $H S T$ F606W-band imaging data assuming a Sérsic model. For systems with multiple lenses, values for the primary lenses are reported. Column 9 is the classification with codes denoting the foreground-lens morphology, the foreground-lens multiplicity, and the status of system as a lens based on available data. Morphology is coded by "E" for early-type (elliptical and S0) and "L" for late-type (Sa and later). Multiplicity is coded by "S" for single and "M" for multiple. Lens status is coded by "A" for systems with clear and convincing evidence of multiple imaging, " $\mathrm{M}$ " for systems with possible evidence of multiple imaging, and " $\mathrm{X}$ " for non-lenses.

hence bias the fitting process. Therefore, we perform the foreground-light subtraction jointly with the lens modeling, as done in Shu et al. (2016b) and Rusu et al. (2016).

In particular, we use the elliptical Sérsic profile to model the foreground light in the joint modeling. Note that lfit_gui also offers two other commonly used profiles, the core-Sérsic profile (Graham et al. 2003; Trujillo et al. 2004) and the Hernquist profile (Hernquist 1990), as models for the foreground light. To investigate the impact of the joint modeling approach, we perform lens modeling on the b-spline-subtracted residual image as well. The results are compared in Section 3.2.

\subsubsection{Lens Mass Model}

Following our previous works (Bolton et al. 2008a; Brownstein et al. 2012; Shu et al. 2015, 2016b), the mass distribution of the foreground lens is modeled with the singular isothermal ellipsoid (SIE) model, which has a projected twodimensional surface mass density profile of

$$
\Sigma(x, y)=\Sigma_{\text {crit }} \frac{\sqrt{q}}{2} \frac{b_{\mathrm{SIE}}}{\sqrt{x^{2}+q^{2} y^{2}}}
$$

where $\Sigma_{\text {crit }}$ is the critical density determined by the cosmological distances as

$$
\Sigma_{\text {crit }}=\frac{c^{2}}{4 \pi G} \frac{d_{S}}{d_{L} d_{L S}}
$$

and $d_{L}, d_{S}$, and $d_{L S}$ are the angular diameter distances from the observer to the lens, from the observer to the source, and between the lens and the source, respectively. The lensing strength of the SIE model $b_{\mathrm{SIE}}$ is equivalent to the Einstein radius in the "intermediate axis" convention for elliptical models, and $q$ is the minor-to-major axis ratio of the isodensity contours. We use one or two SIE components depending on the foreground-lens multiplicity.

We further include an external shear for three systems residing in crowded environments for which the pure SIE models fail to yield good fits. The effective lensing potential of the external shear is

$$
\psi_{\text {shear }}(r, \phi)=-\frac{\gamma}{2} r^{2} \cos 2\left(\phi-\phi_{\gamma}\right)
$$

in which $\gamma$ and $\phi_{\gamma}$ are the strength and position angle of the external shear. Note that singular power-law ellipsoid (Tessore \& Metcalf 2015), spherical Navarro-Frenk-White (Bartelmann 

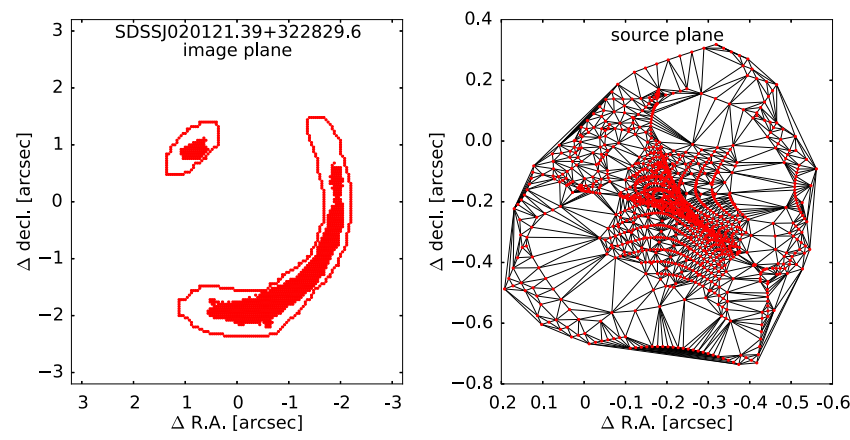

Figure 2. Illustration of the irregular source grid construction from a Delaunay tessellation for the grade-A lens J020121.39+322829.6. The left panel shows the pixels (red dots) in the image plane that are used to construct the Delaunay tessellation, while the right panel shows the corresponding positions (red dots) in the source plane after the mapping. The black lines outline the resulting Delaunay triangles.

1996; Golse \& Kneib 2002), and point mass lens models are also provided in lfit_gui.

\subsubsection{Source-light Model}

The surface brightness distribution of the background source is reconstructed parametrically using elliptical Sérsic models. Because the morphologies of the lensed LAEs are highly irregular and clumpy, multiple Sérsic components are usually needed to recover the observed lensing features. We therefore employ a pixelized source model as a guide to determine the number of required Sérsic components. We obtain a first guess of the model parameters by considering only one Sérsic component, which is able to capture the most significant features in the observational data. We then keep the lens model parameters fixed and generate a pixelized source model based on which extra Sérsic components are added. This is done iteratively until the parametric source model and the pixelized source model are in reasonable agreement.

The pixelized source model is obtained through the semilinear inversion method first introduced by Warren \& Dye (2003). Following the later development of this technique (Dye \& Warren 2005; Koopmans 2005; Brewer \& Lewis 2006; Suyu et al. 2006; Vegetti \& Koopmans 2009; Nightingale \& Dye 2015), we adopt an irregular source grid constructed from the Delaunay tessellation for a set of $N$ points in the source plane that are directly mapped from $N$ pixels in the image plane through the lens equation given a particular lens model. The convolution with the PSF is incorporated as part of the process. The $N$ pixels in the image plane are chosen to contain the $N_{b}$ pixels on the boundaries of the feature masks and the brightest $N-N_{b}$ pixels within the feature masks. The number $N$ is chosen to be equal to half of the total pixel number within the feature masks. The inclusion of the $N_{b}$ boundary pixels ensures that any pixel within the feature masks will be mapped inside the Delaunay tessellation. We choose to use the brightest $N-N_{b}$ pixels in the image plane to preserve as many of the high significance features as possible. Additional linear regularization is included to regulate the overall smoothness of the pixelized source (Warren \& Dye 2003).

Figure 2 illustrates this source gridding process using the grade-A lens system J020121.39+322829.6 as an example. The feature masks for J020121.39+322829.6 enclose 2728 pixels in total. The left panel in Figure 2 shows in red dots the 370 pixels on the boundaries of the feature masks and the brightest $2728 / 2-370=994$ pixels within the feature masks. We then map those 1364 pixels in the image plane back to the source plane for a given set of lens parameters, which are the red dots in the right panel. The black lines resulting from the Delaunay tessellation connect the 1364 pixels in the source plane. The remaining 1364 pixels in the image plane will then be mapped into individual Delaunay triangles, the weights of which are assumed to be inversely proportional to the distances to the three vertices of the triangles that they reside in.

\subsubsection{Optimization}

The parameter optimization is done by minimizing a $\chi^{2}$ function using the Levenberg-Marquardt algorithm as implemented in the LMFIT package (Newville et al. 2014). Each parameter can be assigned a value (required), upper and lower bounds (optional), variability (optional), and a mathematical expression connecting different parameters (optional). For modeling the BELLS GALLERY lenses, the $\chi^{2}$ function is defined as

$$
\chi^{2}=\sum_{i, j} \frac{\left[I_{i, j}^{\mathrm{data}}-\left(I_{i, j}^{\mathrm{phot}}+I_{i, j}^{\mathrm{image}}\right) * \mathrm{PSF}\right]^{2}}{\sigma_{i, j}^{2}},
$$

where the asterisk represents a convolution. $I_{i, j}^{\mathrm{data}}, I_{i, j}^{\mathrm{phot}}$, and $I_{i, j}^{\text {image }}$ are the observed, foreground-light, and model lensed image intensities at pixel $(i, j)$ in the image plane, respectively, and $\sigma_{i, j}$ is the corresponding rescaled pixel count error.

Note that lfit_gui also offers another mode for the parameter optimization, which is a fully Bayesian method utilizing the Affine Invariant Markov Chain Monte Carlo Ensemble sampler (Goodman \& Weare 2010) implemented in the emcee package (Foreman-Mackey et al. 2013). We choose to use the nonlinear Levenberg-Marquardt approach in this work because it is sufficient for the purpose of obtaining smooth lens models and it significantly reduces the computational time when compared to the MCMC approach. Nevertheless, the statistical uncertainties estimated from the covariance matrix in the nonlinear approach are typically underestimated, and the MCMC approach is needed for a better estimate of the parameter uncertainties.

\subsection{Results}

Table 2 provides the lens model parameters of the 17 BELLS GALLERY grade-A lenses obtained from this nonlinear optimization approach. Thirteen systems can be well explained by a simple SIE lens model. Substantial amounts of external shear are also required for J075523.52+344539.5, J091859.21 +510452.5 , and $\mathrm{J} 122656.45+545739.0$ which are also seen to reside in crowded environments. J011300.57+025046.2 and J091807.86+451856.7 each require the inclusion of a second SIE mass component to model the gravitational effects from nearby luminous perturbers. Detailed descriptions of these five systems are given in Table 3.

The best-fit lens parameters for the two foreground-light subtraction schemes are almost identical for 13 of the 17 grade-A lenses. The four systems with significantly larger relative deviations in the Einstein radius are J023740.63064112.9 (3.0\%), J085621.59+201040.5 (3.0\%), J111634.55 $+091503.0(1.8 \%)$, and $\mathrm{J} 222825.76+120503.9$ (1.9\%). These four systems turn out to have relatively smaller Einstein radii and similar two image configurations with one image very 
Table 2

Lens Parameters of the BELLS GALLERY Grade-A Lenses

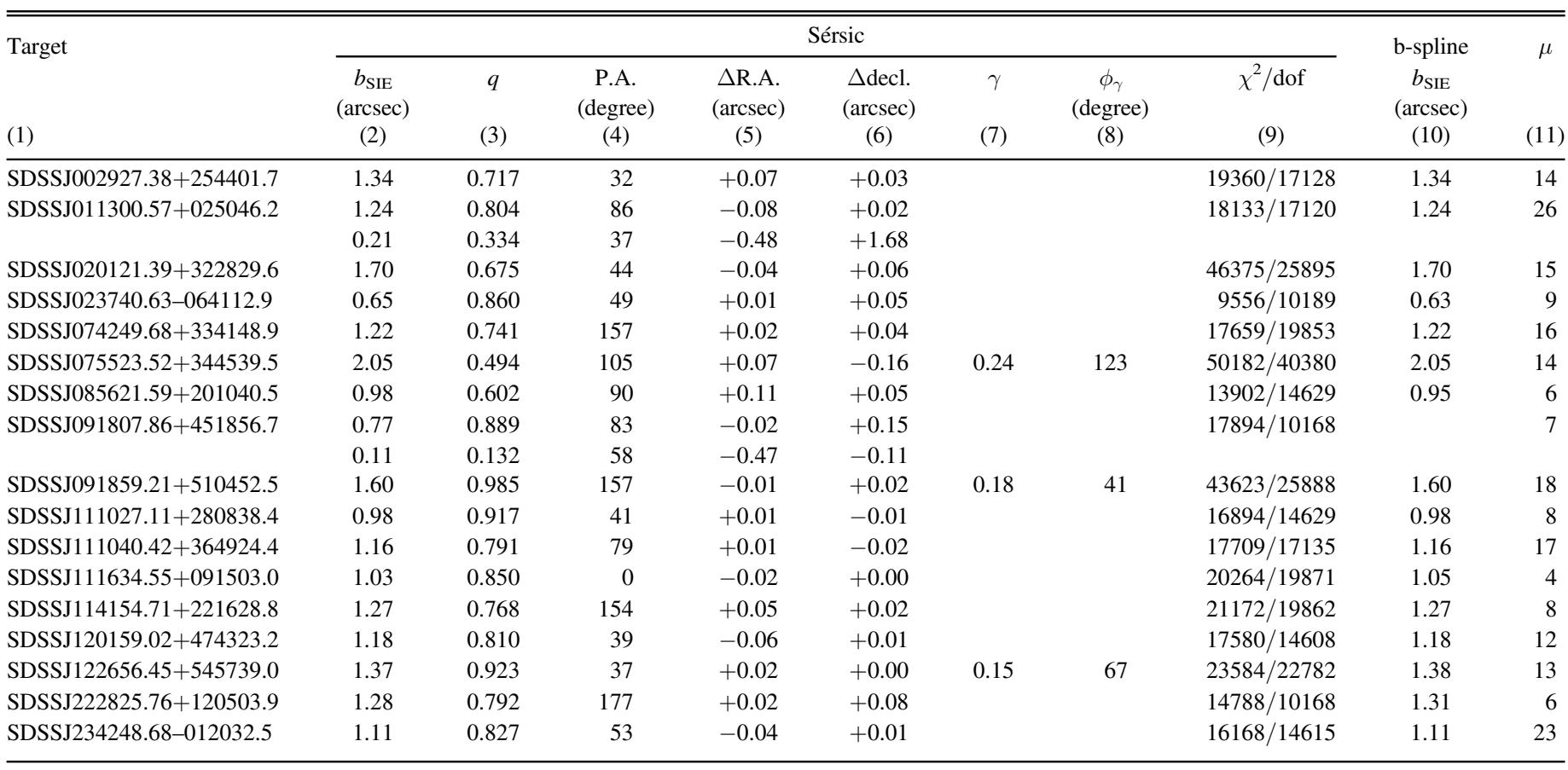

Note. Column 1 is the SDSS system name. Columns 2-8 are the Einstein radius, minor-to-major axis ratio, major-axis position angle with respect to the north, centroid offset in R.A. and decl. of the SIE component, and strength and polar angle of the external shear found using the Sérsic model for foreground-light subtraction. Column 9 provides the $\chi^{2}$ value and the number of degrees of freedom (dof). Column 10 is the Einstein radius found using the b-spline model for foreground-light subtraction. Column 11 is the average magnification found using the Sérsic model for foreground-light subtraction. For SDSSJ011300.57+025046.2 and SDSSJ091807.86+451856.7, the second row provides the parameters of the second SIE component.

Table 3

Notes on Five Grade-A Lenses with Special Treatments

\begin{tabular}{ll}
\hline \hline Target & Comments \\
\hline SDSSJ011300.57+025046.2 & The arc near the location of the bright galaxy to the north of the lens galaxy is bent slightly inward. \\
A second SIE mass component is used to model this perturber. Although another bright galaxy is seen \\
to the south of the lens galaxy, the inclusion of a third SIE component does not yield a significantly better fit. \\
A quadruple-image plus a double-image system. Several nearby luminous clumps are clearly seen. External \\
shear is needed to correctly model the positions of the quadruple images. The inferred mass center is \\
offset from the light center by 0 "' 17 , which is suspected to be the result of the local environment.
\end{tabular}

close to the lens galaxy. This likely makes the results more sensitive to the method used to subtract the unlensed light. Consequently, we only report results using the joint modeling approach. The center of the SIE component is allowed to vary during the lens modeling. In most cases, the inferred lens position is consistent with the observed position of the lens galaxies given the typical model position uncertainties of 0 ".04 (see Columns 5 and 6 of Table 2). Two systems,
$\mathrm{J} 075523.52+344539.5$ and J091807.86+451856.7, show significant spatial offsets $(>0$ !' 12) between the mass and light and are discussed further in Table 3. Because the source and lensed images are spatially extended, we define the average magnification to be the ratio of the observed total light of the lensed images to that of the source. The background LAEs are magnified by factors from 4 to 26 with a median magnification of 13 . 
Dust extinction, especially differential extinction, in the lens galaxy could affect the lens modeling result. In addition, imperfect foreground-light subtraction may also introduce an equivalent effect. Therefore, we perform a simple test on the effects of differential extinction on the inferred SIE parameters using mock lenses with a double-image configuration. More specifically, we generate a double-image lens system with one image very close to the lens galaxy (analogous to J085621.59 +201040.5 ). Then we perform the same lens modeling on this mock lens with the close-in image gradually dimmed, and compare the recovered parameter values to the input values. We find that for this mock lens, the Einstein radius is still well recovered with $\sim 7 \%$ accuracy for a differential extinction of up to $1 \mathrm{mag}$. The SIE mass center shifts toward the close-in image in order to match its effectively lower magnification. The shift can reach as large as 0 " $05-0$ !" 2 for a differential extinction of 0.5 mag.

Although we cannot determine the dust (differential) extinction levels for the BELLS GALLERY lens systems given the single-band $H S T$ imaging observations, a study by Elíasdóttir et al. (2006) shows that the mean differential extinction for their full lensing galaxy sample is only $0.33 \pm 0.03 \mathrm{mag}$, a level that will not affect the fitting parameters significantly according to our test. In addition, our lenses are all massive ETGs, so we expect the amount of differential extinction to be relatively small (e.g., Falco et al. 1999; Kochanek et al. 1999; Elíasdóttir et al. 2006; Vegetti et al. 2012; More et al. 2016).

Table 4 provides the best-fit parameters for each individual source component for the 17 BELLS GALLERY grade-A lenses. The quoted uncertainty in the effective radius is the statistical uncertainty, and the uncertainty in the apparent source magnitude is a combination in quadrature of the statistical uncertainty and a 0.1 mag systematic uncertainty due to foreground subtraction as suggested in Marshall et al. (2007) and Shu et al. (2016b). Note that some source components are not well constrained with large uncertainties. An MCMC approach is demanded for a better source structure recovery. Most of the systems require multiple source components with typical projected separations of $500 \mathrm{pc}-$ $2 \mathrm{kpc}$. The effective radii of the source components are as small as $0 . \prime 0037$, or equivalently $31 \mathrm{pc}$. The best-fit magnificationcorrected, rest-frame, far-UV, apparent $\mathrm{AB}$ magnitudes of the source components range from 29.6 to 24.2. Such resolution and sensitivity cannot be achieved without the aid of the average $\sim 13 \times$ lensing magnification. As a comparison, direct observations of unlensed LAEs at similar redshifts can only reach a UV magnitude limit of 25-27 (e.g., Shapley et al. 2003; Bond et al. 2012; Kobayashi et al. 2016).

Figure 3 displays the results for both the parameterized and pixelized source models for the 17 grade-A lenses. For most of the lenses, smooth models obtained with the nonlinear fitting approach are able to explain the observational data down to the noise level, and the reduced $\chi^{2}$ values are close to unity (Table 2). There are bright residuals with significances greater than $10 \sigma$ in some lenses (e.g., J020121.39+322829.6, $\mathrm{J} 075523.52+344539.5$, and J091859.21+510452.5). These could be a sign for the presence of dark substructures, or caused by other artifacts such as an inappropriate PSF or foreground subtraction. A detailed analysis considering beyond the smooth lens model for these lens systems and the other BELLS GALLERY grade-A lenses is deferred to a future paper. The parameterized and pixelized source models agree reasonably well. The regularization level for the pixelized source model is chosen such that the resulting $\chi^{2}$ value is comparable to that of the parameterized source model. By design, the pixelized source model is optimized to capture the brightest pixels. Artificial, fragmentary structures are seen toward the lower surface brightness edges.

\section{DISCUSSION}

The lens galaxies in the BELLS GALLERY sample are, by selection, similar in many ways to those in the BELLS sample. Figure 4 compares some of the properties of the two samples. In particular, the lens redshift distributions are similar, and the typical sizes of the lens galaxies in the two samples as characterized by the median effective radii are 0"'98 (BELLS GALLERY) and 1"'00 (BELLS), respectively. On the other hand, the BELLS GALLERY lenses are relatively more massive than BELLS lenses with an average stellar velocity dispersion of $272 \mathrm{~km} \mathrm{~s}^{-1}$ as compared to $208 \mathrm{~km} \mathrm{~s}^{-1}$. Combined with the higher source redshifts of the BELLS GALLERY sample, the Einstein radii of the BELLS GALLERY lenses are generally larger. The median Einstein radius of the 17 BELLS GALLERY grade-A lenses is 1 !'22, while the median Einstein radius of the 25 BELLS grade-A lenses is 0!"75 (Brownstein et al. 2012). Consequently, the ratio of Einstein radius to effective radius for the BELLS GALLERY grade-A lenses has a median of 1.37 , and $41 \%(7 / 17)$ of the ratios are larger than two. These numbers are 0.80 and $4 \%$ (1/ 25 ) for the BELLS grade-A lenses. As strong lensing provides an accurate estimate of the total mass within the Einstein radius, a joint analysis of the BELLS and BELLS GALLERY lenses will strongly constrain the radius evolution of the mass profile in massive ETGs (e.g., Rusin et al. 2003; Oguri et al. 2014).

The scientific motivations of the BELLS GALLERY survey are to search for dark substructures within galaxies and extend the mass detection threshold using the intrinsically compact, high-redshift LAEs. As suggested by Figure 3, smooth (SIE plus external shear) lens models only accounting for the luminous components can already explain the observational data of most of the lens systems well. However, this only indicates the absence of substructures near the LOS to the lensed images with masses high enough to generate visually observable signals. In fact, previous dark substructure detections based on the lensed image surface brightness data are mostly confirmed statistically by comparing Bayesian evidences (e.g., Vegetti et al. 2010, 2012; Fadely \& Keeton 2012; Hezaveh et al. 2016). Thus, the smooth lens models presented in this paper are only an important first step in searching for dark substructures in the BELLS GALLERY lens sample. Further investigations under a Bayesian framework are deferred to future papers.

Besides the lens galaxies, the lensed sources-high-redshift LAEs - are of great interest. LAEs are believed to be young, low-mass, and highly star-forming galaxies. They compose an important piece in our understanding of galaxy evolution scenarios (e.g., Venemans et al. 2005; Bond et al. 2012; Ciardullo et al. 2012; Ao et al. 2015; Finkelstein et al. 2015; Hathi et al. 2016; Song et al. 2016; Zheng et al. 2016). They can also be used as a probe of high-redshift circumgalactic and interstellar media (e.g., Miralda-Escudé \& Rees 1998; Malhotra \& Rhoads 2004; Zheng et al. 2010, 2011b; Zheng \& 
Table 4

Source Parameters of the BELLS GALLERY Grade-A Lenses

\begin{tabular}{|c|c|c|c|c|c|c|c|c|c|}
\hline Target & $\begin{array}{l}\text { Source } \\
\text { ID } \\
\text { (2) }\end{array}$ & $\begin{array}{c}\Delta \text { R.A. } \\
\text { (arcsec) } \\
\text { (3) }\end{array}$ & $\begin{array}{l}\Delta \text { Decl. } \\
(\operatorname{arcsec}) \\
(4)\end{array}$ & (5) & (6) & $\begin{array}{c}R_{\text {eff }} \\
(\operatorname{arcsec}) \\
(7)\end{array}$ & $\begin{array}{l}R_{\text {eff }} \\
(\mathrm{pc}) \\
(8)\end{array}$ & $\begin{array}{l}\Delta d \\
(\mathrm{pc}) \\
(9)\end{array}$ & $\begin{array}{c}m_{\mathrm{AB}} \\
(\mathrm{mag}) \\
(10)\end{array}$ \\
\hline \multirow[t]{4}{*}{ SDSSJ002927.38+254401.7 } & S1 & -0.08 & -0.10 & 0.08 & 0.33 & $0.0062 \pm 0.0011$ & $51 \pm 8$ & 0 & $25.3 \pm 1.2$ \\
\hline & S2 & -0.16 & +0.12 & 0.52 & 4.02 & $0.2931 \pm 0.1163$ & $2435 \pm 966$ & 1944 & $25.6 \pm 1.4$ \\
\hline & S3 & -0.07 & -0.11 & 0.59 & 1.57 & $0.0173 \pm 0.0011$ & $144 \pm 8$ & 94 & $26.1 \pm 0.6$ \\
\hline & S4 & -0.13 & -0.09 & 0.15 & 3.00 & $0.0640 \pm 0.0125$ & $531 \pm 103$ & 413 & $27.3 \pm 0.9$ \\
\hline \multirow[t]{2}{*}{ SDSSJ011300.57+025046.2 } & S1 & -0.03 & +0.19 & 0.57 & 3.10 & $0.1267 \pm 0.0341$ & $1039 \pm 279$ & 0 & $27.1 \pm 0.7$ \\
\hline & S2 & -0.27 & +0.15 & 0.13 & 1.40 & $0.0543 \pm 0.0041$ & $445 \pm 33$ & 2002 & $27.1 \pm 0.3$ \\
\hline \multirow[t]{3}{*}{ SDSSJ020121.39+322829.6 } & S1 & +0.25 & -0.25 & 0.17 & 4.66 & $0.1326 \pm 0.0112$ & $1066 \pm 89$ & 0 & $24.8 \pm 0.2$ \\
\hline & S2 & +0.24 & -0.10 & 0.40 & 3.44 & $0.2183 \pm 0.0277$ & $1756 \pm 223$ & 1202 & $24.5 \pm 0.5$ \\
\hline & S3 & +0.21 & -0.30 & 0.72 & 0.18 & $0.1020 \pm 0.0007$ & $821 \pm 5$ & 558 & $24.8 \pm 0.1$ \\
\hline SDSSJ023740.63-064112.9 & S1 & -0.08 & +0.01 & 0.46 & 1.46 & $0.0206 \pm 0.0023$ & $174 \pm 19$ & 0 & $27.4 \pm 0.4$ \\
\hline \multirow[t]{3}{*}{ SDSSJ074249.68+334148.9 } & S1 & -0.22 & +0.15 & 0.17 & 1.68 & $0.0138 \pm 0.0007$ & $115 \pm 6$ & 0 & $27.1 \pm 0.5$ \\
\hline & S2 & -0.17 & +0.10 & 0.30 & 0.05 & $0.0194 \pm 0.5311$ & $162 \pm 4444$ & 658 & $27.3 \pm 0.1$ \\
\hline & S3 & -0.23 & +0.13 & 0.65 & 2.28 & $0.1172 \pm 0.0077$ & $980 \pm 64$ & 179 & $25.6 \pm 0.2$ \\
\hline \multirow[t]{3}{*}{ SDSSJ075523.52+344539.5 } & S1 & +0.07 & -0.36 & 0.52 & 1.07 & $0.1294 \pm 0.0013$ & $1058 \pm 10$ & 0 & $24.5 \pm 0.1$ \\
\hline & S2 & -0.01 & -0.28 & 0.47 & 0.33 & $0.0324 \pm 0.0003$ & $264 \pm 2$ & 999 & $25.5 \pm 0.1$ \\
\hline & S3 & +0.65 & +0.48 & 0.42 & 0.57 & $0.1431 \pm 0.0062$ & $1170 \pm 50$ & 8339 & $26.1 \pm 0.2$ \\
\hline SDSSJ085621.59+201040.5 & S1 & -0.34 & +0.16 & 0.60 & 5.39 & $0.0617 \pm 0.0090$ & $521 \pm 76$ & 0 & $25.5 \pm 2.3$ \\
\hline \multirow{2}{*}{ SDSSJ091807.86+451856.7 } & S1 & +0.06 & -0.02 & 0.66 & 2.32 & $0.0209 \pm 0.0009$ & $175 \pm 7$ & 0 & $25.6 \pm 0.5$ \\
\hline & S2 & +0.27 & +0.01 & 0.37 & 1.78 & $0.3800 \pm 0.0468$ & $3184 \pm 392$ & 1793 & $24.5 \pm 0.2$ \\
\hline \multirow[t]{3}{*}{ SDSSJ091859.21+510452.5 } & S1 & +0.08 & -0.27 & 0.09 & 0.06 & $0.0103 \pm 0.0042$ & $86 \pm 35$ & 0 & $24.9 \pm 0.1$ \\
\hline & S2 & +0.27 & -0.15 & 0.29 & 0.19 & $0.0109 \pm 0.0006$ & $90 \pm 5$ & 1909 & $27.8 \pm 0.1$ \\
\hline & S3 & +0.17 & -0.22 & 0.34 & 1.73 & $0.1478 \pm 0.0044$ & $1232 \pm 36$ & 894 & $24.7 \pm 0.1$ \\
\hline SDSSJ111027.11+280838.4 & S1 & +0.18 & +0.21 & 0.24 & 1.27 & $0.0436 \pm 0.0022$ & $363 \pm 18$ & 0 & $26.2 \pm 0.2$ \\
\hline \multirow{4}{*}{ SDSSJ111040.42+364924.4 } & S1 & -0.02 & -0.07 & 0.65 & 0.52 & $0.0180 \pm 0.0004$ & $148 \pm 3$ & 0 & $27.0 \pm 0.1$ \\
\hline & S2 & -0.03 & -0.23 & 0.44 & 0.54 & $0.0495 \pm 0.0013$ & $410 \pm 10$ & 1278 & $26.6 \pm 0.1$ \\
\hline & S3 & +0.00 & -0.01 & 0.75 & 0.42 & $0.0243 \pm 0.0006$ & $201 \pm 5$ & 582 & $27.6 \pm 0.1$ \\
\hline & S4 & +0.03 & -0.10 & 0.87 & 2.29 & $0.3174 \pm 0.0495$ & $2626 \pm 409$ & 421 & $25.2 \pm 0.2$ \\
\hline \multirow[t]{2}{*}{ SDSSJ111634.55+091503.0 } & S1 & +0.05 & -0.61 & 0.17 & 0.05 & $0.0259 \pm 0.1889$ & $215 \pm 1569$ & 0 & $25.1 \pm 0.1$ \\
\hline & S2 & -0.01 & -0.55 & 0.64 & 0.90 & $0.1008 \pm 0.0057$ & $837 \pm 47$ & 748 & $25.5 \pm 0.1$ \\
\hline \multirow[t]{2}{*}{ SDSSJ114154.71+221628.8 } & S1 & -0.25 & -0.03 & 0.79 & 4.72 & $0.0298 \pm 0.0018$ & $240 \pm 14$ & 0 & $24.9 \pm 1.2$ \\
\hline & S2 & -0.21 & +0.07 & 0.49 & 0.05 & $0.0195 \pm 0.1626$ & $157 \pm 1315$ & 882 & $27.8 \pm 0.1$ \\
\hline \multirow[t]{4}{*}{ SDSSJ120159.02+474323.2 } & S1 & +0.02 & +0.11 & 0.79 & 4.47 & $0.2796 \pm 0.0432$ & $2378 \pm 367$ & 0 & $24.2 \pm 0.6$ \\
\hline & S2 & +0.11 & +0.03 & 0.46 & 0.05 & $0.0269 \pm 1.9713$ & $228 \pm 16768$ & 1038 & $26.6 \pm 0.1$ \\
\hline & S3 & -0.23 & +0.28 & 0.56 & 1.31 & $0.0970 \pm 0.0089$ & $825 \pm 75$ & 2554 & $26.5 \pm 0.2$ \\
\hline & S4 & +0.03 & +0.09 & 0.04 & 0.28 & $0.0037 \pm 0.0002$ & $31 \pm 1$ & 185 & $29.6 \pm 0.2$ \\
\hline \multirow[t]{4}{*}{ SDSSJ122656.45+545739.0 } & S1 & -0.09 & -0.10 & 0.41 & 13.21 & $2.7357 \pm 5.3906$ & $22190 \pm 43725$ & 0 & $24.7 \pm 9.1$ \\
\hline & S2 & +0.18 & +0.00 & 0.67 & 5.07 & $0.0135 \pm 0.0021$ & $109 \pm 17$ & 2358 & $26.9 \pm 3.6$ \\
\hline & S3 & -0.17 & +0.01 & 0.37 & 0.48 & $0.0447 \pm 0.0019$ & $362 \pm 15$ & 1181 & $27.8 \pm 0.2$ \\
\hline & S4 & -0.05 & -0.13 & 0.19 & 0.36 & $0.0033 \pm 0.0339$ & $27 \pm 275$ & 353 & $21.2 \pm 123.4$ \\
\hline \multirow[t]{2}{*}{ SDSSJ222825.76+120503.9 } & S1 & +0.26 & -0.13 & 0.41 & 1.80 & $0.0077 \pm 0.0045$ & $61 \pm 35$ & 0 & $25.5 \pm 3.7$ \\
\hline & S2 & +0.30 & -0.25 & 0.61 & 3.13 & $0.2470 \pm 0.0627$ & $1985 \pm 504$ & 956 & $25.5 \pm 0.9$ \\
\hline \multirow[t]{3}{*}{ SDSSJ234248.68-012032.5 } & S1 & -0.07 & -0.09 & 0.62 & 2.26 & $0.0998 \pm 0.0117$ & $841 \pm 98$ & 0 & $26.4 \pm 0.4$ \\
\hline & S2 & -0.04 & -0.05 & 0.49 & 0.05 & $0.0058 \pm 0.0321$ & $49 \pm 270$ & 465 & $28.5 \pm 0.1$ \\
\hline & S3 & -0.15 & -0.23 & 0.50 & 0.05 & $0.0198 \pm 0.4625$ & $167 \pm 3898$ & 1302 & $28.5 \pm 0.2$ \\
\hline
\end{tabular}

Note. Column 1 is the SDSS system name. Columns 2-8 provide ID, central positions relative to the center of the cutout in R.A. and decl., minor-to-major axis ratio, Sérsic index, effective radius in arcsec, and effective radius in parsecs of each individual source component. Column 9 is the projected separation from the first source component in parsecs. Column 10 is the magnification-corrected rest-frame far-UV apparent AB magnitude of each individual source component calculated from the best-fit source model. 

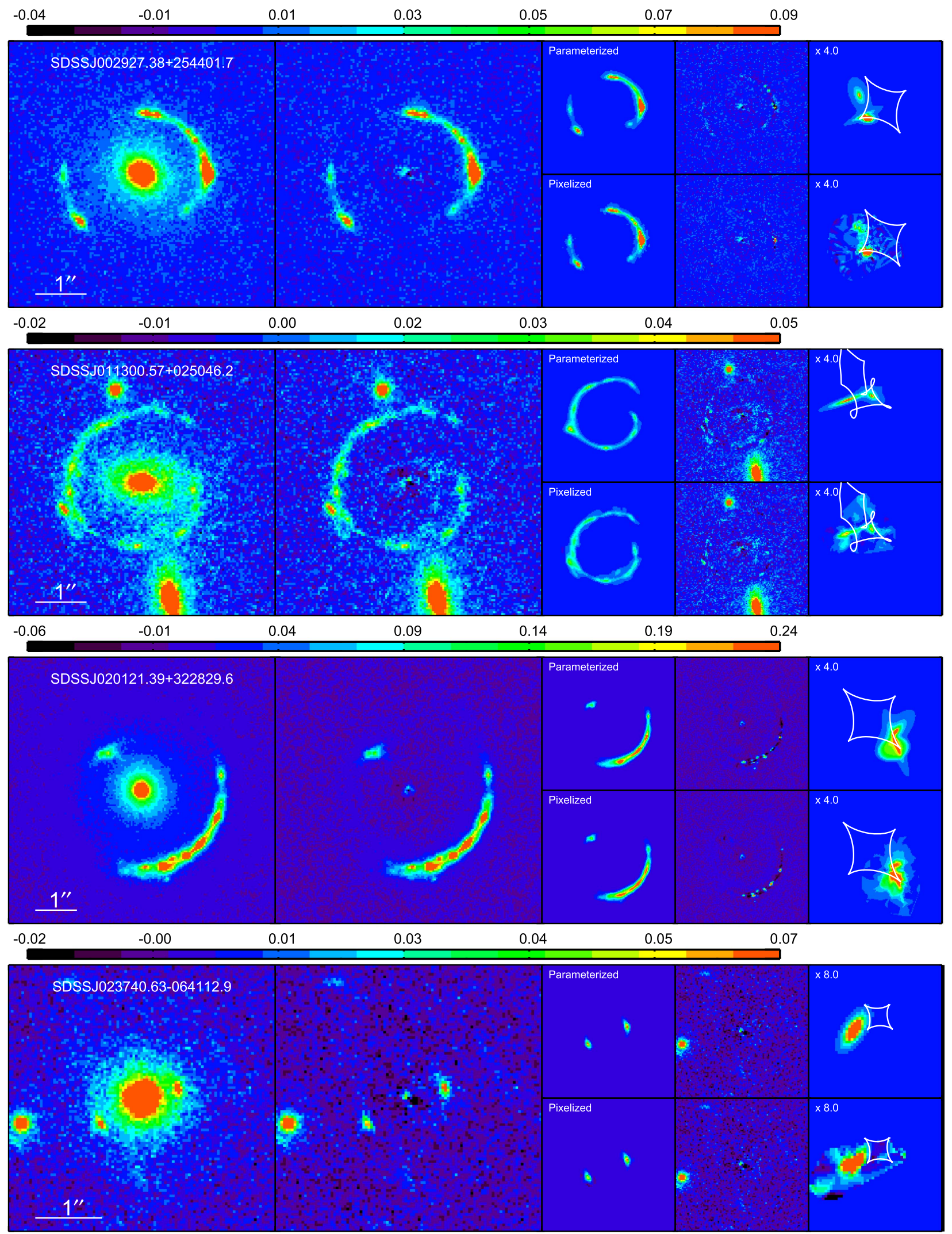

Figure 3. Smooth lens models for the 17 BELLS GALLERY grade-A lenses. The observational data, foreground-subtracted image, predicted lensed image, final residual, and the background source model are shown from left to right, respectively. For each system, the results of the two source models are split into two rows with the parameterized source model on the top and the pixelized source model on the bottom. The white lines in the last panels are the caustics of the lens model. All the images are orientated such that north is up and east is to the left. The source plane panels are magnified by factors of four or eight relative to the image plane panel as indicated in each panel. The color bars indicate the intensity levels in units of electrons per second per pixel ${ }^{2}$. 

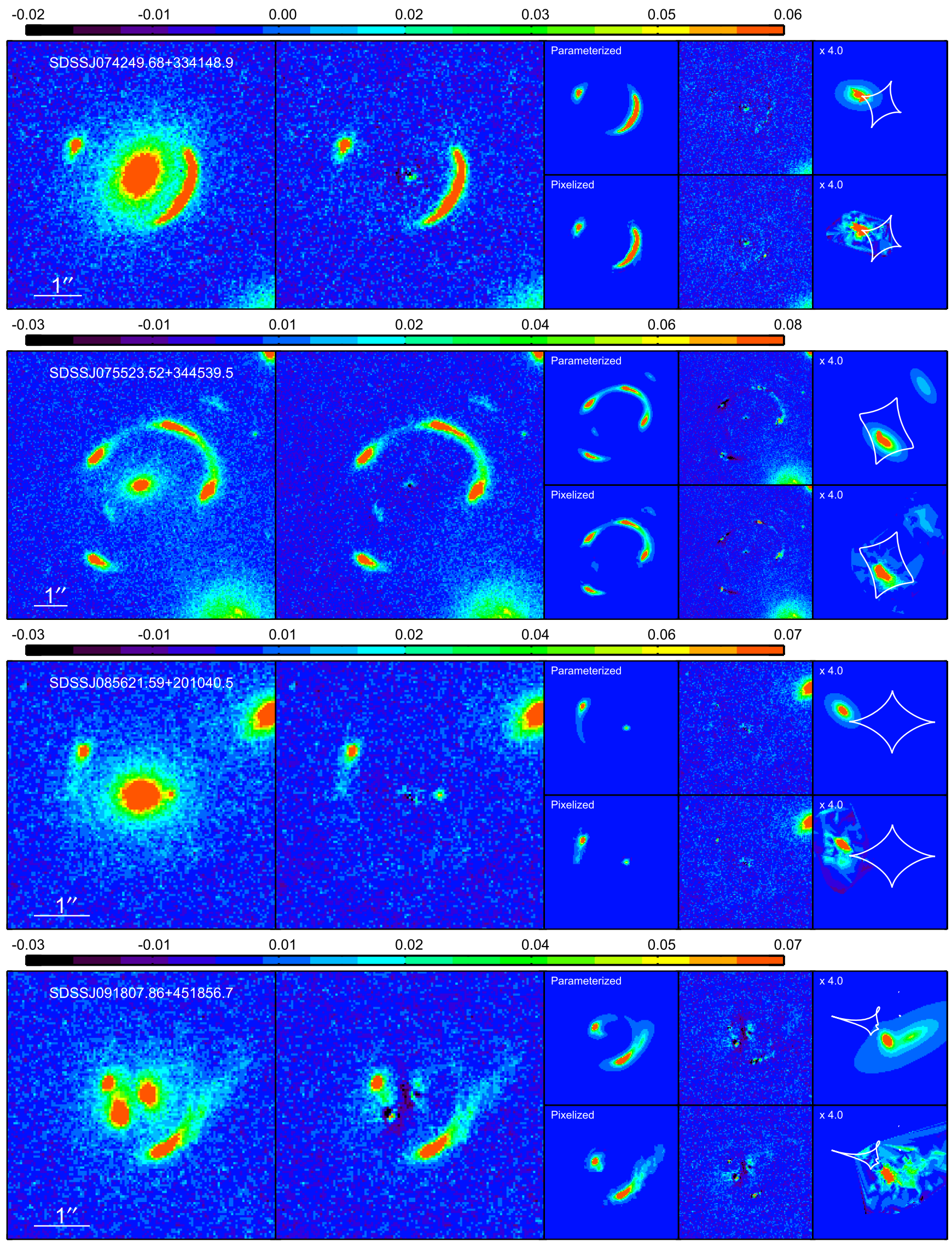

Figure 3. (Continued.) 

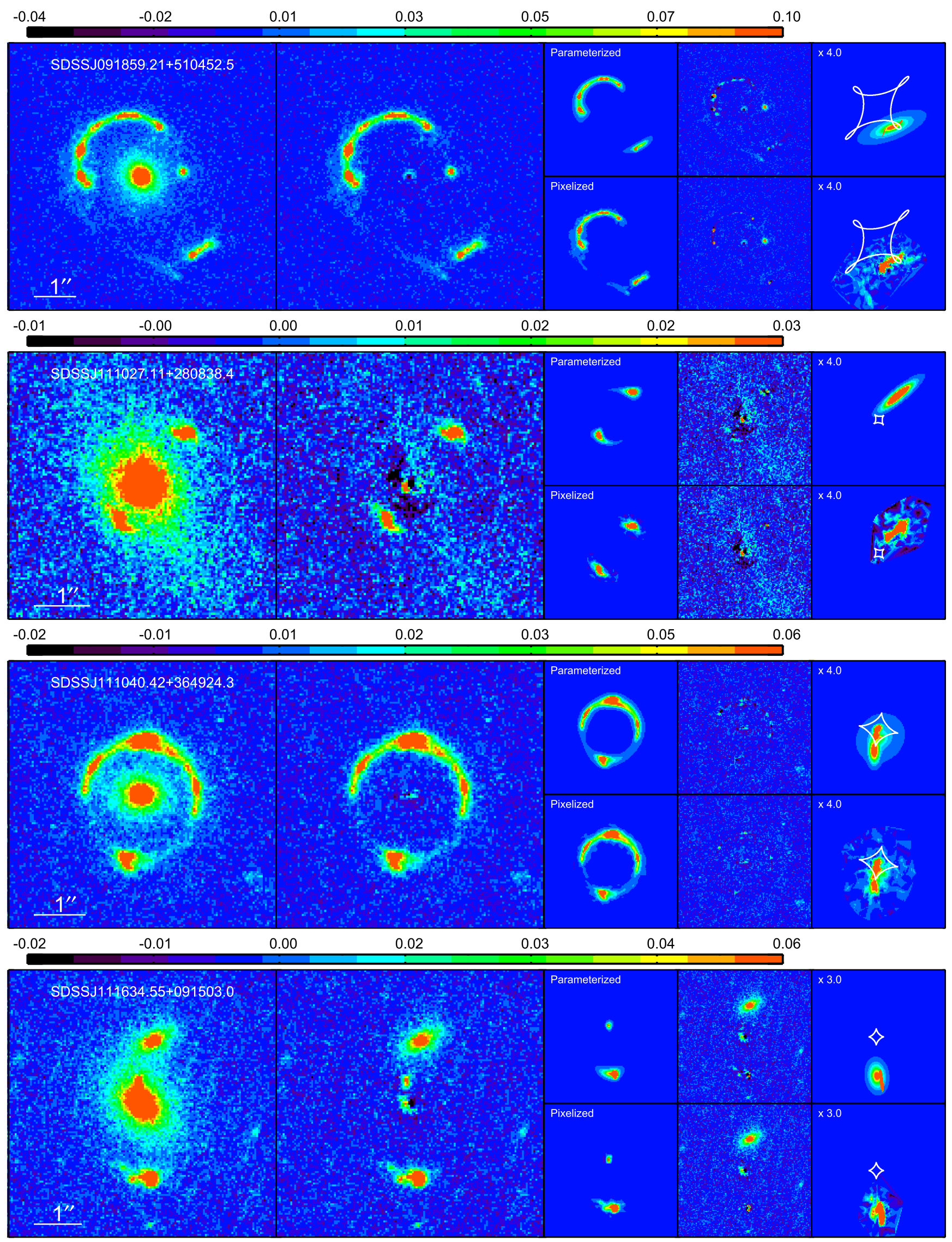

Figure 3. (Continued.) 


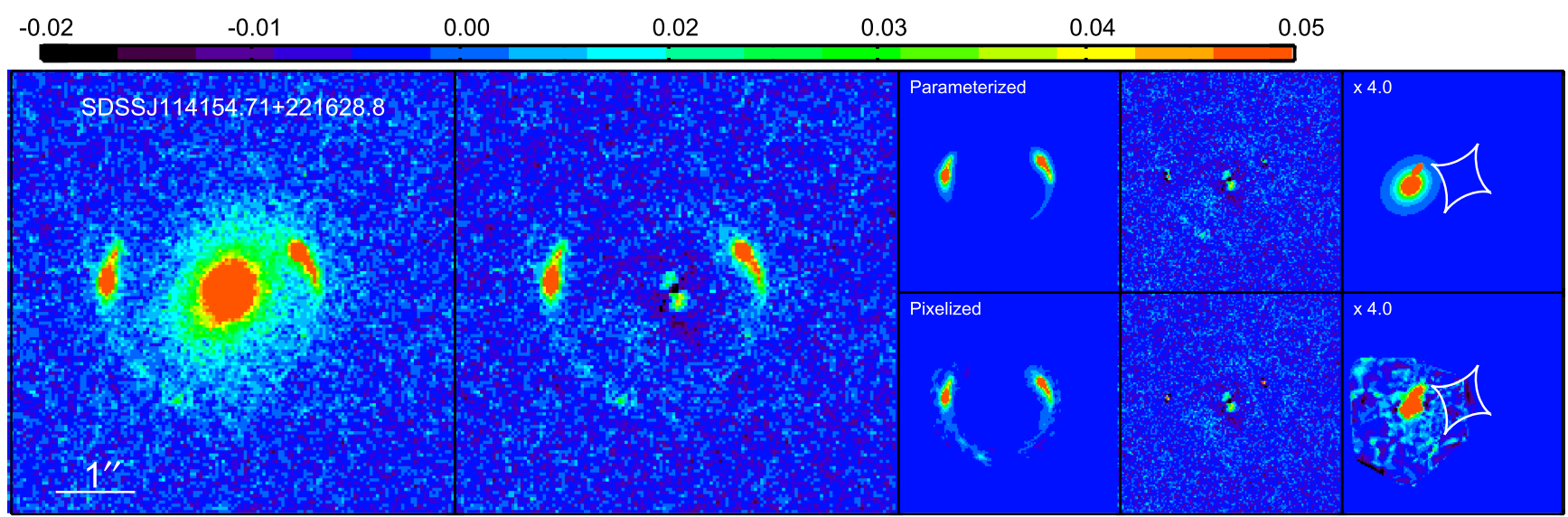

\begin{tabular}{lllllll}
-0.03 & -0.01 & 0.01 & 0.03 & 0.04 & 0.06 & 0.08 \\
\hline
\end{tabular}


Figure 3. (Continued.) 


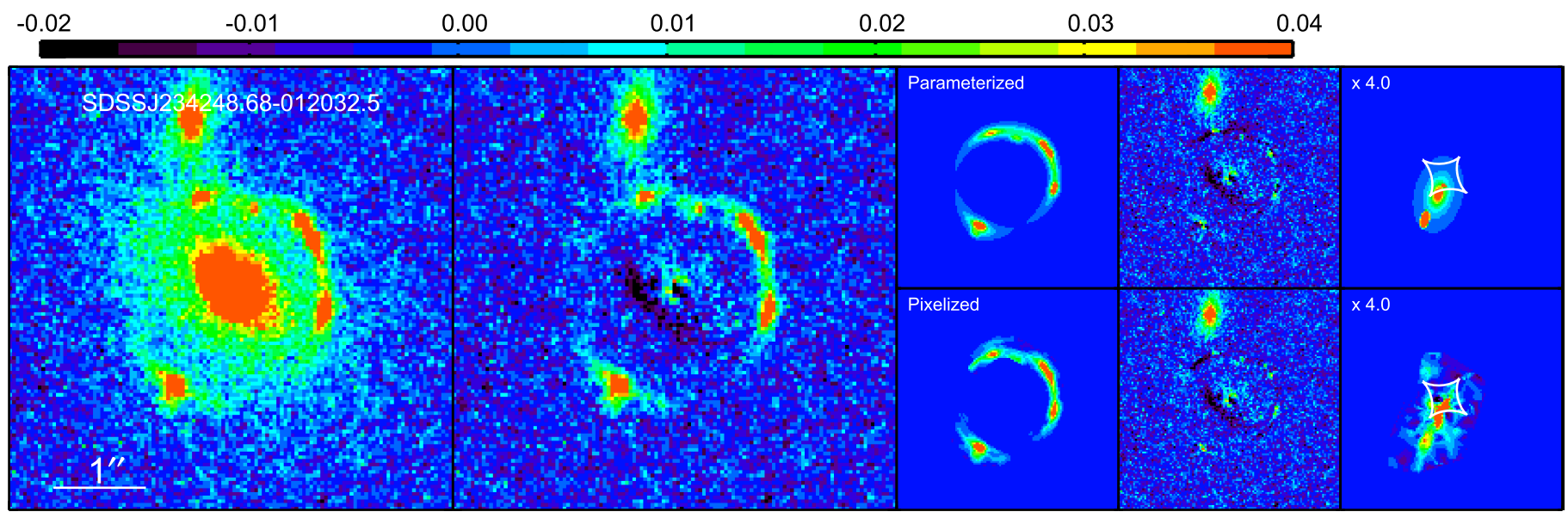

Figure 3. (Continued.)
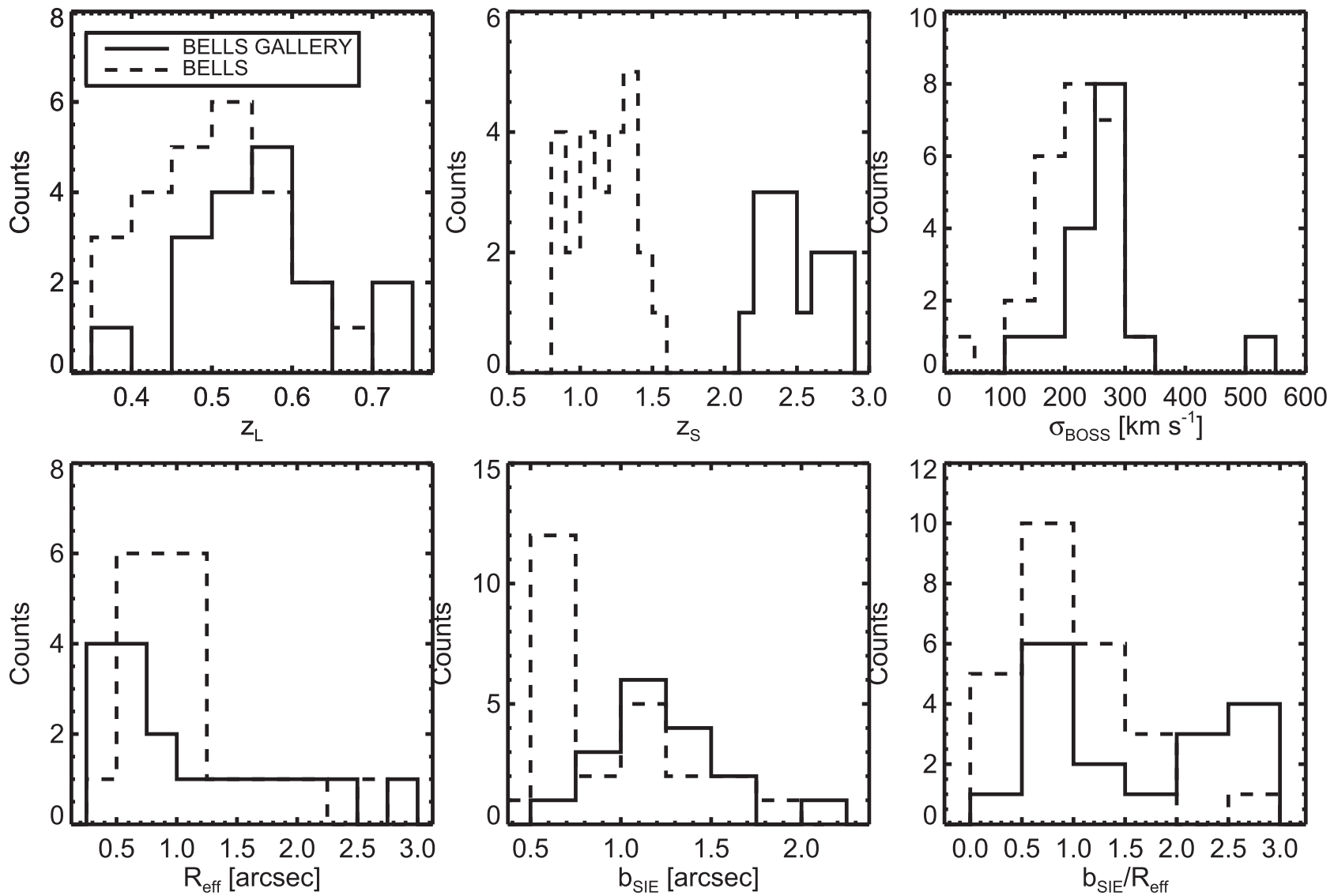

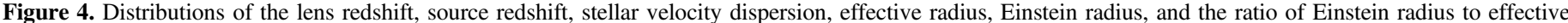
radius for the BELLS (dashed histograms) and the BELLS GALLERY (solid histograms) lens samples.

Wallace 2014) and as tracers of high-redshift large-scale structures (e.g., Hill et al. 2008; Tamura et al. 2009; Zheng et al. 2011a). In particular, the HST F606W filter covers the rest-frame far-ultraviolet (UV) emission from the background LAEs. Previous studies of unlensed LAEs over a wide redshift range $\left(0.03<z_{\mathrm{LAE}}<7.0\right)$ have shown that their far-UV morphologies are sometimes $(\sim 20 \%-50 \%)$ clumpy and/or irregular as a result of in situ or merger-triggered star formation (e.g., Bond et al. 2009, 2012; Jiang et al. 2013; Hayes et al. 2014; Guaita et al. 2015; Kobayashi et al. 2016).
However, as we have discussed in Shu et al. (2016a), direct observations of LAEs are limited to the most luminous examples and any explorations of their "fine" structures on sub-kiloparsec scales are challenging and sometimes impossible. As shown in Table 4 and Figure 3, by combining the superb angular resolution of $H S T$ with the average $\sim 13 \times$ magnification of these lenses, we are able to resolve individual, faint star-forming knots in LAEs at redshifts from two to three smaller than $\sim 100 \mathrm{pc}$. A detailed study of the properties of the lensed LAEs and comparisons with their unlensed 
counterparts are deferred to a forthcoming paper. Furthermore, infrared and sub-millimeter spectroscopic follow-up observations of the highly magnified BELLS GALLERY lens sample targeted at the rest-frame atomic and molecular lines in the LAEs will permit exploration of the interstellar medium (ISM) and circumgalactic medium (CGM) of these high-redshift galaxies.

\section{CONCLUSIONS}

We present HST WFC3 F606W-band imaging observations of 21 galaxy-Ly $\alpha$ strong gravitational lens candidates. The sample, known as the BELLS GALLERY sample, was spectroscopically selected from almost 1.5 million galaxy spectra in the final data release of the BOSS survey in SDSSIII. The foreground-lens galaxies are at a typical redshift of 0.55 and the $\operatorname{Ly} \alpha$ emission comes from redshifts from two to three.

The HST data are fully reduced and analyzed by the custombuilt tools ACSPROC and lfit_gui, respectively. After modeling the systems with smooth lens models consisting of SIE mass distributions for the luminous components and an external shear, the main findings are as follows.

1. Seventeen systems are confirmed to be grade-A lenses including 8 with extended arcs, 3 with quadruple images, and 6 with double images. Three systems are singly imaged non-lenses, while the remaining system is a temporary "maybe" with complex structures that are hard to interpret based solely on single-band data. Considering the typical $50 \%$ success rate in the previous surveys with similar selection techniques, another $\sim 70$ galaxy-LAE strong lenses are expected among the remaining 166 lens candidates in the parent sample.

2. We demonstrate that different foreground-light subtraction schemes can lead to different model parameter estimations particularly for two image systems where the fractional differences in the inferred Einstein radii are $2 \%-3 \%$. This result highlights the need of performing foreground-light subtraction jointly with the lens modeling.

3. Because of the much higher source redshifts and more massive lens galaxies, the Einstein radii of the BELLS GALLERY lenses are generally larger than those of the BELLS lenses, while the lens galaxy sizes are comparable. As a result, the combination of the BELLS GALLERY and BELLS lenses can constrain any radius evolution of the mass profile in massive ETGs.

4. The smooth lens models seem to be adequate for explaining the observed imaging data for most of the 17 BELLS GALLERY grade-A lenses as shown in Figure 3. Although this suggests the absence of massive dark substructures near the lensing features, a thorough exploration of substructures in a statistical manner is required.

5. The average lensing magnifications of the background LAEs are found to be 4-26. The LAEs are thus resolved into individual star-forming knots of a wide range of properties. They have characteristic sizes from less than $100 \mathrm{pc}$ to several kiloparsecs, rest-frame, far-UV, apparent $\mathrm{AB}$ magnitudes from 29.6 to 24.2, and typical projected separations of $500 \mathrm{pc}$ to $2 \mathrm{kpc}$. Further followup spectroscopic observations will reveal the ISM and CGM properties of these high-redshift galaxies.
We thank the anonymous referee for helpful comments. This work has been partially supported by the Strategic Priority Research Program "The Emergence of Cosmological Structures" of the Chinese Academy of Sciences Grant No. XDB09000000 and by the National Natural Science Foundation of China (NSFC) under grant numbers 11333003, 11390372 (Y.S. and S.M.), and 11603032 (Y.S.). C.S.K. is partially supported by NSF grant AST-1515876. The work of M.O. was supported in part by World Premier International Research Center Initiative (WPI Initiative), MEXT, Japan, and JSPS KAKENHI Grant Number 26800093 and $15 \mathrm{H} 05892$. Z.Z. is partially supported by NASA grant NNX14AC89G and NSF grant AST-1208891. B.M. acknowledges support from NSF-1313302.

Support for program \#14189 was provided by NASA through a grant from the Space Telescope Science Institute, which is operated by the Association of Universities for Research in Astronomy, Inc., under NASA contract NAS 5-26555.

Funding for SDSS-III was provided by the Alfred P. Sloan Foundation, the Participating Institutions, the National Science Foundation, and the U.S. Department of Energy Office of Science. The SDSS-III website is http://www.sdss3.org/.

SDSS-III was managed by the Astrophysical Research Consortium for the Participating Institutions of the SDSS-III Collaboration including the University of Arizona, the Brazilian Participation Group, Brookhaven National Laboratory, Carnegie Mellon University, University of Florida, the French Participation Group, the German Participation Group, Harvard University, the Instituto de Astrofisica de Canarias, the Michigan State/Notre Dame/JINA Participation Group, Johns Hopkins University, Lawrence Berkeley National Laboratory, Max Planck Institute for Astrophysics, Max Planck Institute for Extraterrestrial Physics, New Mexico State University, New York University, Ohio State University, Pennsylvania State University, University of Portsmouth, Princeton University, the Spanish Participation Group, University of Tokyo, University of Utah, Vanderbilt University, University of Virginia, University of Washington, and Yale University.

\section{REFERENCES}

Ao, Y., Matsuda, Y., Beelen, A., et al. 2015, A\&A, 581, A132

Auger, M. W., Treu, T., Bolton, A. S., et al. 2009, ApJ, 705, 1099

Auger, M. W., Treu, T., Bolton, A. S., et al. 2010, ApJ, 724, 511

Barnabè, M., Czoske, O., Koopmans, L. V. E., et al. 2009, MNRAS, 399, 21 Barnabè, M., Czoske, O., Koopmans, L. V. E., Treu, T., \& Bolton, A. S. 2011, MNRAS, 415, 2215

Barnabè, M., Dutton, A. A., Marshall, P. J., et al. 2012, MNRAS, 423, 1073 Bartelmann, M. 1996, A\&A, 313, 697

Bolton, A. S., Brownstein, J. R., Kochanek, C. S., et al. 2012b, ApJ, 757, 82 Bolton, A. S., Burles, S., Koopmans, L. V. E., et al. 2008a, ApJ, 682, 964

Bolton, A. S., Burles, S., Koopmans, L. V. E., Treu, T., \& Moustakas, L. A. 2006a, ApJ, 638, 703

Bolton, A. S., Burles, S., Schlegel, D. J., Eisenstein, D. J., \& Brinkmann, J. 2004, AJ, 127, 1860

Bolton, A. S., Moustakas, L. A., Stern, D., et al. 2006b, ApJL, 646, L45 Bolton, A. S., Schlegel, D. J., Aubourg, É., et al. 2012a, AJ, 144, 144 Bolton, A. S., Treu, T., Koopmans, L. V. E., et al. 2008b, ApJ, 684, 248 Bond, N. A., Gawiser, E., Gronwall, C., et al. 2009, ApJ, 705, 639 Bond, N. A., Gawiser, E., Guaita, L., et al. 2012, ApJ, 753, 95 Brewer, B. J., Dutton, A. A., Treu, T., et al. 2012, MNRAS, 422, 3574 Brewer, B. J., \& Lewis, G. F. 2006, ApJ, 637, 608

Brewer, B. J., Marshall, P. J., Auger, M. W., et al. 2014, MNRAS, 437, 1950 Brownstein, J. R., Bolton, A. S., Schlegel, D. J., et al. 2012, ApJ, 744, 41 Bussmann, R. S., Pérez-Fournon, I., Amber, S., et al. 2013, ApJ, 779, 25 Christensen, L., Richard, J., Hjorth, J., et al. 2012, MNRAS, 427, 1953 
Ciardullo, R., Gronwall, C., Wolf, C., et al. 2012, ApJ, 744, 110

Czoske, O., Barnabè, M., Koopmans, L. V. E., Treu, T., \& Bolton, A. S. 2008, MNRAS, 384, 987

Dawson, K. S., Schlegel, D. J., Ahn, C. P., et al. 2013, AJ, 145, 10

Dutton, A. A., Brewer, B. J., Marshall, P. J., et al. 2011, MNRAS, 417, 1621

Dutton, A. A., Treu, T., Brewer, B. J., et al. 2013, MNRAS, 428, 3183

Dye, S., \& Warren, S. J. 2005, ApJ, 623, 31

Eisenstein, D. J., Weinberg, D. H., Agol, E., et al. 2011, AJ, 142, 72

Elíasdóttir, Á., Hjorth, J., Toft, S., Burud, I., \& Paraficz, D. 2006, ApJS, 166, 443

Fadely, R., \& Keeton, C. R. 2012, MNRAS, 419, 936

Falco, E. E., Impey, C. D., Kochanek, C. S., et al. 1999, ApJ, 523, 617

Finkelstein, K. D., Finkelstein, S. L., Tilvi, V., et al. 2015, ApJ, 813, 78

Foreman-Mackey, D., Hogg, D. W., Lang, D., \& Goodman, J. 2013, PASP, 125,306

Gavazzi, R., Treu, T., Koopmans, L. V. E., et al. 2008, ApJ, 677, 1046

Gavazzi, R., Treu, T., Rhodes, J. D., et al. 2007, ApJ, 667, 176

Golse, G., \& Kneib, J.-P. 2002, A\&A, 390, 821

Goodman, J., \& Weare, J. 2010, Comm. App. Math. Comp. Sci., 5, 65

Graham, A. W., Erwin, P., Trujillo, I., \& Asensio Ramos, A. 2003, AJ, 125,2951

Grillo, C., Eichner, T., Seitz, S., et al. 2010, ApJ, 710, 372

Guaita, L., Melinder, J., Hayes, M., et al. 2015, A\&A, 576, A51

Hao, C. N., Mao, S., Deng, Z. G., Xia, X. Y., \& Wu, H. 2006, MNRAS, 370, 1339

Hathi, N. P., Le Fèvre, O., Ilbert, O., et al. 2016, A\&A, 588, A26

Hayes, M., Östlin, G., Duval, F., et al. 2014, ApJ, 782, 6

Hernquist, L. 1990, ApJ, 356, 359

Hezaveh, Y. D., Dalal, N., Marrone, D. P., et al. 2016, ApJ, 823, 37

Hill, G. J., Gebhardt, K., Komatsu, E., et al. 2008, in ASP Conf. Ser. 399, Panoramic Views of Galaxy Formation and Evolution, ed. T. Kodama, T. Yamada, \& K. Aoki (San Francisco, CA: ASP), 115

Inoue, K. T., Minezaki, T., Matsushita, S., \& Chiba, M. 2016, MNRAS, 457, 2936

Jiang, L., Egami, E., Fan, X., et al. 2013, ApJ, 773, 153

Karman, W., Grillo, C., Balestra, I., et al. 2016, A\&A, 585, A27

Keeton, C. R., Kochanek, C. S., \& Falco, E. E. 1998, ApJ, 509, 561

Kobayashi, M. A. R., Murata, K. L., Koekemoer, A. M., et al. 2016, ApJ, 819, 25

Kochanek, C. S. 1995, ApJ, 445, 559

Kochanek, C. S., Falco, E. E., Impey, C. D., et al. 1999, in AIP Conf. Ser. 470, After the Dark Ages: When Galaxies were Young, ed. S. Holt \& E. Smith (Melville, NY: AIP), 163

Komatsu, E., Smith, K. M., Dunkley, J., et al. 2011, ApJS, 192, 18

Koopmans, L. V. E. 2005, MNRAS, 363, 1136

Koopmans, L. V. E., Treu, T., Bolton, A. S., Burles, S., \& Moustakas, L. A. 2006, ApJ, 649, 599

Krist, J. 1993, in ASP Conf. Ser. 52, Astronomical Data Analysis Software and Systems II, ed. R. J. Hanisch, R. J. V. Brissenden, \& J. Barnes (San Francisco, CA: ASP), 536

MacLeod, C. L., Jones, R., Agol, E., \& Kochanek, C. S. 2013, ApJ, 773, 35

Malhotra, S., \& Rhoads, J. E. 2004, ApJL, 617, L5

Marshall, P. J., Treu, T., Melbourne, J., et al. 2007, ApJ, 671, 1196

Miralda-Escudé, J., \& Rees, M. J. 1998, ApJ, 497, 21

More, A., Lee, C.-H., Oguri, M., et al. 2016, MNRAS, submitted (arXiv:1608. 06288)
Moustakas, L. A., Marshall, P., Newman, J. A., et al. 2007, ApJL, 660, L31

Muzzin, A., Labbé, I., Franx, M., et al. 2012, ApJ, 761, 142

Newton, E. R., Marshall, P. J., Treu, T., et al. 2011, ApJ, 734, 104

Newville, M., Stensitzki, T., Allen, D. B., \& Ingargiola, A. 2014, LMFIT: NonLinear Least-Square Minimization and Curve-Fitting for Python, Zenodo, doi: $10.5281 /$ zenodo. 11813

Nierenberg, A. M., Treu, T., Wright, S. A., Fassnacht, C. D., \& Auger, M. W. 2014, MNRAS, 442, 2434

Nightingale, J. W., \& Dye, S. 2015, MNRAS, 452, 2940

Oguri, M., Rusu, C. E., \& Falco, E. E. 2014, MNRAS, 439, 2494

Quider, A. M., Pettini, M., Shapley, A. E., \& Steidel, C. C. 2009, MNRAS, 398, 1263

Rusin, D., Kochanek, C. S., \& Keeton, C. R. 2003, ApJ, 595, 29

Rusu, C. E., Oguri, M., Minowa, Y., et al. 2016, MNRAS, 458, 2

Sérsic, J. L. 1963, BAAA, 6, 41

Shapley, A. E., Steidel, C. C., Pettini, M., \& Adelberger, K. L. 2003, ApJ, 588,65

Shu, Y., Bolton, A. S., Brownstein, J. R., et al. 2015, ApJ, 803, 71

Shu, Y., Bolton, A. S., Kochanek, C. S., et al. 2016a, ApJ, 824, 86

Shu, Y., Bolton, A. S., Moustakas, L. A., et al. 2016b, ApJ, 820, 43

Shu, Y., Bolton, A. S., Schlegel, D. J., et al. 2012, AJ, 143, 90

Song, M., Finkelstein, S. L., Livermore, R. C., et al. 2016, ApJ, in press (arXiv:1602.02160)

Sonnenfeld, A., Gavazzi, R., Suyu, S. H., Treu, T., \& Marshall, P. J. 2013, ApJ, 777, 97

Spilker, J., Marrone, D., Aravena, M., et al. 2016, ApJ, 826, 112

Spiniello, C., Koopmans, L. V. E., Trager, S. C., Czoske, O., \& Treu, T. 2011, MNRAS, 417, 3000

Stark, D. P., Walth, G., Charlot, S., et al. 2015, MNRAS, 454, 1393

Suyu, S. H., \& Halkola, A. 2010, A\&A, 524, A94

Suyu, S. H., Marshall, P. J., Hobson, M. P., \& Blandford, R. D. 2006, MNRAS, 371, 983

Tamura, Y., Kohno, K., Nakanishi, K., et al. 2009, Natur, 459, 61

Tessore, N., \& Metcalf, R. B. 2015, A\&A, 580, A79

Treu, T., Dutton, A. A., Auger, M. W., et al. 2011, MNRAS, 417, 1601

Treu, T., Gavazzi, R., Gorecki, A., et al. 2009, ApJ, 690, 670

Treu, T., Koopmans, L. V., Bolton, A. S., Burles, S., \& Moustakas, L. A. 2006, ApJ, 640, 662

Trujillo, I., Erwin, P., Asensio Ramos, A., \& Graham, A. W. 2004, AJ, 127,1917

Vegetti, S., \& Koopmans, L. V. E. 2009, MNRAS, 392, 945

Vegetti, S., Koopmans, L. V. E., Bolton, A., Treu, T., \& Gavazzi, R. 2010, MNRAS, 408, 1969

Vegetti, S., Lagattuta, D. J., McKean, J. P., et al. 2012, Natur, 481, 341

Venemans, B. P., Röttgering, H. J. A., Miley, G. K., et al. 2005, A\&A, 431,793

Warren, S. J., \& Dye, S. 2003, ApJ, 590, 673

York, D. G., Adelman, J., Anderson, J. E., Jr., et al. 2000, AJ, 120, 1579

Zheng, Z., Cen, R., Trac, H., \& Miralda-Escudé, J. 2010, ApJ, 716, 574

Zheng, Z., Cen, R., Trac, H., \& Miralda-Escudé, J. 2011a, ApJ, 726, 38

Zheng, Z., Cen, R., Weinberg, D., Trac, H., \& Miralda-Escudé, J. 2011b, ApJ, 739,62

Zheng, Z., \& Wallace, J. 2014, ApJ, 794, 116

Zheng, Z.-Y., Malhotra, S., Rhoads, J. E., et al. 2016, ApJS, 226, 23 